\title{
Oscillating nuclear electric dipole moment induced by axion dark matter produces atomic and molecular electric dipole moments and nuclear spin rotation
}

\author{
V. V. Flambaum \\ School of Physics, University of New South Wales, Sydney 2052, Australia, \\ Helmholtz Institute Mainz, Johannes Gutenberg-Universität Mainz, 55099 Mainz, Germany, \\ and The New Zealand Institute for Advanced Study, Massey University Auckland, \\ 0632 Auckland, New Zealand \\ H. B. Tran Tan $\odot$ \\ School of Physics, University of New South Wales, Sydney 2052, Australia
}

(Received 16 April 2019; published 27 December 2019)

\begin{abstract}
According to the Schiff theorem, a static nuclear electric dipole moment (EDM) does not produce atomic and molecular EDMs. However, interaction with the axion dark matter field generates nuclear EDMs $\mathbf{d}_{N}=\mathbf{d}_{N}^{0} \cos (\omega t)$ which oscillate with the frequency $\omega=m_{a} c^{2} / \hbar$. These oscillating nuclear EDMs generate atomic and molecular EDMs proportional to $\omega^{2}$. The resulting atomic and molecular EDMs do not, however, lead to nuclear spin rotation in constant external electric fields. On the other hand, an oscillating electric field penetrates atoms and molecules and can interact with nuclear EDMs. Moreover, if the nuclear EDM oscillation frequency is in resonance with the external field, nuclear spin rotation happens, with the rotation angle growing linearly with time.
\end{abstract}

DOI: 10.1103/PhysRevD.100.111301

\section{INTRODUCTION}

It was suggested in Ref. [1] that interactions with axionic dark matter produce oscillating neutrons and thus oscillating nuclear electric dipole moments (EDMs). We show that such oscillating EDMs will not be limited by the Schiff theorem [2] and will produce the oscillating atomic and molecular EDMs calculated below. However, a constant electric field does not penetrate to the nucleus so there is no interaction with the nuclear EDM, i.e., there will be no nuclear spin rotation for both static and oscillating nuclear EDMs.

Observable atomic and molecular EDMs are actually produced by the nuclear Schiff moment which is suppressed compared to EDMs by an additional second power of the nuclear radius which is very small on the atomic scale [3-7] (see also Refs. [8-14] for other effects producing atomic and molecular EDMs). The effects produced by the axion-induced Schiff moment have been considered in Ref. [15]. A corresponding experiment in solids has been proposed in Ref. [16]. The first results of the oscillating neutron EDM and a $\mathrm{Hg}$ atom's EDM measurements were

Published by the American Physical Society under the terms of the Creative Commons Attribution 4.0 International license. Further distribution of this work must maintain attribution to the author(s) and the published article's title, journal citation, and DOI. Funded by SCOAP . presented in Ref. [17] where the limits on the low-mass axion interaction constant with matter have been improved by up to 3 orders of magnitude.

In this paper, we consider the situation when both the nuclear EDM and the external field are oscillating. In such cases, the external field penetrates to the atomic and molecular nucleus [18-20] and can interact with the nuclear EDM. We demonstrate that in the case where the frequency of the external field matches the frequency of the nuclear EDM oscillation, nuclear spin rotation happens with the rotation angle growing linearly with time. This effect is, in principle, observable.

We will also present the result for the atomic and molecular EDMs induced by nuclear EDMs. It will be observed that the molecular EDM is larger than its atomic counterpart, due to the fact that nuclei move much slower than electrons so their screening effect is much weaker. As a result, the residual, partly screened EDMs in molecules are $M_{N} / m_{e}$ times larger than those in atoms. Here $M_{N}$ is the nuclear mass and $m_{e}$ is the electron mass. An additional $M_{N} / m_{e}$ appears due to the small energy intervals between rotational states in the molecule.

\section{SCREENING THEOREM FOR TIME- DEPENDENT ELECTRIC FIELDS AND EDMS}

As known, a nucleus in a neutral system (atom or molecule) is completely screened from a constant electric 
field [2]. Here we will present a derivation of this fact following the Appendix in Ref. [21]. For definiteness, we assume that the system in question is a neutral atom in a static homogeneous external electric field of arbitrary strength (we ignore the possibility of atomic ionization and effects of magnetic fields).

The Hamiltonian of an atom placed in a static homogeneous external electric field $\mathbf{E}_{0}$ is

$$
\begin{aligned}
H= & \sum_{i}\left[K_{i}-e \phi_{0}\left(\mathbf{r}_{i}\right)+e \mathbf{r}_{i} \cdot \mathbf{E}_{0}\right] \\
& +\sum_{i>j} \frac{e^{2}}{\left|\mathbf{r}_{i}-\mathbf{r}_{j}\right|}-\mathbf{d} \cdot \mathbf{E}_{0}
\end{aligned}
$$

where $K_{i}$ and $\mathbf{r}_{i}$ are the kinetic energy and coordinates of the electrons, $\mathbf{d}$ is the static nuclear EDM and $\phi_{0}\left(\mathbf{r}_{i}\right)$ is the electrostatic nuclear potential given by

$$
\phi_{0}\left(\mathbf{r}_{i}\right)=e \int \frac{\rho(\mathbf{r}) d^{3} \mathbf{r}}{\left|\mathbf{r}_{i}-\mathbf{r}\right|}
$$

where $\rho$ is the nuclear charge distribution. We consider here the case of an infinitely heavy nucleus. The nuclear recoil correction is not enough to generate an atomic EDM [2].

We add to $H$ an auxiliary term

$$
V=\mathbf{d} \cdot \mathbf{E}_{0}-\frac{1}{Z e} \sum_{i} \mathbf{d} \cdot \nabla_{i} \phi_{0}\left(\mathbf{r}_{i}\right)
$$

which, in the linear approximation in $\mathbf{d}$, does not produce any energy shift, $\langle V\rangle=0$. Indeed, we have

$$
\frac{i}{m}\left[\sum_{i} \mathbf{p}_{i}, H\right]=-e \sum_{i} \nabla_{i} \phi_{0}\left(\mathbf{r}_{i}\right)+Z e \mathbf{E}_{0}
$$

where we have taken into account the fact that the total electron momentum $\sum_{i} \mathbf{p}_{i}$ commutes with the electronelectron interaction term. Using Eq. (3) and the fact that

$$
\frac{i}{m}\left\langle\psi\left|\left[\sum_{i} \mathbf{p}_{i}, H\right]\right| \psi\right\rangle \propto\left(E_{\psi}-E_{\psi}\right)=0
$$

( $\psi$ is the wave function of the Hamiltonian $H$ ), we obtain

$$
\langle V\rangle=\left\langle\mathbf{d} \cdot \mathbf{E}_{0}-\frac{1}{Z e} \sum_{i} \mathbf{d} \cdot \nabla_{i} \phi_{0}\left(\mathbf{r}_{i}\right)\right\rangle=0
$$

To find an EDM one needs to measure a linear energy shift in an external electric field. Since V does not contribute to this shift we can add it to the Hamiltonian

$$
\begin{aligned}
\tilde{H} & \equiv H+V \\
& =\sum_{i}\left[K_{i}-e \phi\left(\mathbf{r}_{i}\right)+e \mathbf{r}_{i} \cdot \mathbf{E}_{0}\right]+\sum_{i>j} \frac{e^{2}}{\left|\mathbf{r}_{i}-\mathbf{r}_{j}\right|},
\end{aligned}
$$

where

$$
\phi\left(\mathbf{r}_{i}\right)=\phi_{0}\left(\mathbf{r}_{i}\right)+\frac{1}{Z e} \mathbf{d} \cdot \nabla_{i} \phi_{0}\left(\mathbf{r}_{i}\right)
$$

Note that the Hamiltonian $\tilde{H}$ does not contain the direct interaction $\mathbf{d} \cdot \mathbf{E}_{0}$ between the nuclear EDM and external field (Schiff theorem). The dipole term is also canceled out in the multipole expansion of $\phi\left(\mathbf{r}_{i}\right)$.

Let us now consider the case where the nuclear EDM is time dependent $\mathbf{d}=\mathbf{d}(t)$. In this case, Eq. (5) becomes

$$
\begin{aligned}
\frac{i}{m}\left\langle\left[\sum_{i} \mathbf{p}_{i}, H\right]\right\rangle & =-\frac{1}{m} \frac{d}{d t}\left\langle\sum_{i} \mathbf{p}_{i}\right\rangle \\
& =\frac{1}{m} \frac{d\left\langle\mathbf{p}_{\text {nuc }}\right\rangle}{d t} \propto \mathbf{d} .
\end{aligned}
$$

Therefore, the contribution due to $\langle V\rangle$ is zero in the first order in $\mathbf{d}$. As a result, just as in the case of a static nuclear $\mathrm{EDM}$, there is no direct interaction between a timedependent nuclear EDM and a static external electric field, hence, no nuclear spin rotation. Indeed, the external electric field does not penetrate to the nucleus (since an atom and its nucleus are not accelerated by a static homogeneous electric field), so the nuclear EDM has nothing to interact with.

Now consider the case of a time-dependent electric field. In this case, we have

$$
\frac{1}{m} \frac{d\left\langle\mathbf{p}_{\mathrm{nuc}}\right\rangle}{d t} \propto \mathbf{E}_{0}
$$

since the external field now penetrates to the nucleus [18-20]. Indeed, the external electric field forces the electron shells to oscillate and since the atom's center of mass stays at rest, the nucleus must move, so the electric field on it is not zero. Therefore, the nuclear EDM interacts with this electric field and nuclear spin rotation happens.

Note that the absence of nuclear spin rotation in the case of a static electric field does not mean that the oscillating nuclear EDM does not produce any effect. An oscillating nuclear EDM excites the electrons and produces atomic and molecular EDMs (as demonstrated below). This effect is particularly clear in the case where the nuclear EDM's frequency of oscillation is in resonance with some atomic or molecular frequency, in which case the electronic wave function is a linear combination of two states of opposite parities and thus gives rise to oscillating atomic and molecular EDMs. Oscillating nuclear EDMs may also be 
detected using the atomic and molecular transitions they induce, as investigated in Refs. [22-24].

The case where both the nuclear EDM and the external electric field are time dependent, particularly when they are oscillating, is of special interest. As demonstrated in Refs. [18-20], an external electric field which oscillates with a frequency $\Omega, \mathbf{E}_{0} \sim \cos \Omega t$, induces an electric field on the nucleus which oscillates with the same frequency. The interaction of this field with a nuclear EDM which itself oscillates with a frequency $\omega, \mathbf{d}_{N} \sim \cos \omega t$, is proportional to $\cos \omega t \cos \Omega t$. If $\omega=\Omega$ then this interaction contains a time-independent component and the nuclear spin rotation angle grows linearly with time (see below).

\section{NUCLEAR EDMS PRODUCED BY THE AXION DARK MATTER FIELD}

It has been noted in Ref. [25] that the neutron EDM may be produced by the QCD $\theta$ term. Numerous references and recent results for the neutron and proton EDMs were summarized in Ref. [26]:

$$
\begin{aligned}
& d_{n}=-(2.7 \pm 1.2) \times 10^{-16} \theta e \mathrm{~cm} \\
& d_{p}=(2.1 \pm 1.2) \times 10^{-16} \theta e \mathrm{~cm}
\end{aligned}
$$

Calculations of the nuclear EDM produced by the $P$ - and $T$-odd nuclear forces have been performed in Refs. [5-7, 27]. For a general estimate of the nuclear EDM it is convenient to use a single-valence-nucleon formula from Ref. [5] and express the result in terms of $\theta$ following Ref. [28]:

$$
d \approx e\left(q-\frac{Z}{A}\right)(1-2 q) \xi\langle\sigma\rangle
$$

where $\xi=7 \times 10^{-16} \theta \mathrm{cm}$.

Here $q=1$ for the valence proton, $q=0$ for the valence neutron, the nuclear spin matrix element $\langle\sigma\rangle=1$ if $I=$ $l+1 / 2$ and $\langle\sigma\rangle=-I /(I+1)$ if $I=l-1 / 2$. Here, $I$ and $l$ are the total and orbital momenta of the valence nucleon.

It was noted in Ref. [1] that the axion dark matter field may be an oscillating $\theta$ term and thus may generate the oscillating neutron EDM. To reproduce the density of dark matter, following Ref. [15], we may substitute $\theta(t)=$ $\theta_{0} \cos (\omega t)$ where $\theta_{0}=4 \times 10^{-18}, \omega=m_{a} c^{2} / \hbar$ and $m_{a}$ is the axion mass.

\section{EVOLUTION OF AN OSCILLATING NUCLEAR EDM IN AN OSCILLATING ELECTRIC FIELD}

In this section, we investigate the behavior of an oscillating nuclear EDM $\mathbf{d}_{N} \cos \omega t$ when an oscillating external electric field $\mathbf{E}_{0} \cos \Omega t$ is applied to an atom. It was shown in Refs. [18-20] that the field $\mathbf{E}_{0} \cos \Omega t$ induces on the nucleus an electric field of frequency $\Omega$ and amplitude

$$
\mathbf{E}_{N}^{\text {off-res }}=-\frac{\Omega^{2} m_{e} \alpha(\Omega)}{Z e^{2} \hbar^{2}} \mathbf{E}_{0},
$$

where $\alpha$ is the atomic polarizability.

This result applies in the case where $\Omega$ is far away from any atomic transition frequency. In the case where $\Omega$ matches the transition frequency $\omega_{n 0}$ from the ground state $|0\rangle$ to some state $|n\rangle, \mathbf{E}_{N}$ is resonantly enhanced

$$
\mathbf{E}_{N}^{\text {on-res }}=-\frac{2 m_{e}}{\hbar e^{2} Z} \frac{\Omega^{2}}{\Gamma}\left|\left\langle 0\left|D_{z}\right| n\right\rangle\right|^{2} \mathbf{E}_{0},
$$

where $\Gamma$ is the width of the state $|n\rangle$ and $D_{z}$ is the atom's electric dipole operator. Typically, $\Gamma \ll \omega_{n 0}=\Omega$ so $E_{N}^{\text {off-res }} \ll E_{N}^{\text {on-res }}$.

In any case, the interaction of the nuclear EDM $\mathbf{d}_{N} \cos \omega t$ with the field $\mathbf{E}_{N} \cos \Omega t$ may be written as

$$
\begin{aligned}
V= & -\mathbf{d}_{N} \cdot \mathbf{E}_{N} \cos \Omega t \cos \omega t \\
= & -\frac{\mathbf{d}_{N} \cdot \mathbf{E}_{N}}{2} \cos (\Omega+\omega) t \\
& -\frac{\mathbf{d}_{N} \cdot \mathbf{E}_{N}}{2} \cos (\Omega-\omega) t .
\end{aligned}
$$

The evolution of the nuclear spin I under the influence of this interaction (without magnetic fields) is

$$
\begin{aligned}
\langle\mathbf{I}\rangle= & \langle\mathbf{I}\rangle_{0}+d_{N} \operatorname{Im}\left\langle\mathbf{I}\left[\mathbf{I} \cdot \mathbf{E}_{N}\right]\right\rangle_{0} \frac{\sin (\Omega+\omega) t}{\Omega+\omega} \\
& +d_{N} \operatorname{Im}\left\langle\mathbf{I}\left[\mathbf{I} \cdot \mathbf{E}_{N}\right]\right\rangle_{0} \frac{\sin (\Omega-\omega) t}{\Omega-\omega},
\end{aligned}
$$

where $\langle.\rangle_{0}$ means the expectation value with respect to the original state of the nuclear spin.

If $\Omega, \omega \neq 0$ then the second term in Eq. (16) is sinusoidal and thus averages to zero. However, in the case where $\omega=\Omega$, the third term in Eq. (16) is proportional to $t$.

If we assume that the nucleus carries spin $1 / 2$, which initially points in the $x$ direction and that $\mathbf{E}_{0}=E_{0} \hat{\mathbf{z}}$ then in the case where $\Omega=\omega$, Eq. (16) reads (neglecting the oscillating term)

$$
\langle\mathbf{I}\rangle=\frac{1}{2} \hat{\mathbf{x}}-\frac{1}{4} d_{N} E_{N} t \hat{\mathbf{y}},
$$

which shows that $\mathbf{I}$ starts to rotate in the $x y$ plane and the rotation angle is $\theta \sim d_{N} E_{N} t / 2$. In fact, since the result (16) was obtained using perturbation theory, it only holds, in the case where $\Omega=\omega$, if $t$ is small. For large $t$, we should write (neglecting the oscillating term)

$$
\langle\mathbf{I}\rangle=\frac{1}{2} \cos \frac{d_{N} E_{N} t}{2} \hat{\mathbf{x}}-\frac{1}{2} \sin \frac{d_{N} E_{N} t}{2} \hat{\mathbf{y}} .
$$

Note that $E_{N}$ in Eq. (18) may take the off-resonance value (13) or the resonance value (14). The latter is of 
particular interest since in that case $E_{N}$ is enhanced by several orders of magnitude. This situation requires $\Omega=\omega=\omega_{n 0}$. When such a "double resonance" condition is met, nuclear spin rotation may be much more significant.

Note that although the analysis of this section was performed for an atom, the same conclusion holds if we consider a molecule. In fact, molecules have several advantages over atoms. In molecules, nuclei also participate in the screening of an external electric field and since nuclei move much slower than electrons, their ability to screen an oscillating electric field is weaker. As a result, the induced field on a molecular nucleus is enhanced by a factor of $M_{N} / m_{e} \lesssim 10^{5}$ in comparison with the field on an atomic nucleus. A further enhancement to the field on a molecular nucleus comes from the small energy intervals between rotational levels in molecules which appear in the denominator [20]. Finally, since molecules have rich spectra with small energy intervals which are in the expected range of the axion mass, the "double resonance" condition may be more easily satisfied in molecules than in atoms. Achieving this entails tuning the energy interval between the states $|0\rangle$ and $|n\rangle$ (using Stark and Zeeman shifts) and the frequency of the external electric field to match the nuclear EDM oscillation frequency.

\section{OSCILLATING ATOMIC EDMS INDUCED BY OSCILLATING NUCLEAR EDMS}

As remarked earlier, an oscillating nuclear EDM $\mathbf{d}_{N} \cos \omega t$ induces oscillating atomic EDMs. In this section, we provide the calculation for the atomic EDM induced by a nuclear EDM.

The Hamiltonian of an atom in the field of an oscillating nuclear EDM $\mathbf{d}=\mathbf{d}_{0} \cos (\omega t)$ may be written as

$$
V=e \sum_{k=1}^{N_{e}} \frac{\mathbf{d} \cdot \mathbf{r}_{k}}{r_{k}^{3}}=\frac{i}{Z e \hbar}\left[\mathbf{P} \cdot \mathbf{d}, H_{0}\right],
$$

where $H_{0}$ is the Schrödinger or Dirac Hamiltonian for the atomic electrons in the absence of $\mathbf{d}, N_{e}$ is the number of electrons, $Z e$ is the nuclear charge, $Z_{i}=Z-N_{e},-e$ is the electron charge, $r_{k}$ is the electron position relative to the nucleus, and $\mathbf{P}=\sum_{k=1}^{N_{e}} \mathbf{p}_{\mathbf{k}}$ is the total momentum of all atomic electrons (which commutes with the electronelectron interaction but not with the nuclear-electron interaction $\quad U=-\sum_{k=1}^{N_{e}} Z e^{2} / r_{k}: \quad\left[\mathbf{P}, H_{0}\right]=[\mathbf{P}, U]=$ $\left.-i \hbar Z e^{2} \sum_{k=1}^{N_{e}} \nabla \frac{1}{r_{k}}\right)$. Here we assumed that the nuclear mass is infinite and neglected the very small effects of the Breit and magnetic interactions.

Using $H_{0}|n\rangle=E_{n}|n\rangle$ we obtain the matrix element of $V$ between atomic states $|n\rangle$ and $|m\rangle$

$$
\langle n|V| m\rangle=\frac{i E_{n m}}{Z e \hbar}\langle n|\mathbf{P} \cdot \mathbf{d}| m\rangle,
$$

where $E_{n m}=E_{n}-E_{m}$.
Using the time-dependent perturbation theory [29] for the oscillating perturbation $V=V_{0} \cos \omega t$ and Eq. (20) we obtain a formula for the induced atomic EDM

$$
\begin{aligned}
\mathbf{D}_{\text {ind }} & =2 \sum_{n} \frac{E_{0 n} \operatorname{Re}(\langle 0|V| n\rangle\langle n|\mathbf{D}| 0\rangle)}{E_{0 n}^{2}-\epsilon^{2}} \\
& =\frac{2}{Z e \hbar} \sum_{n} \frac{E_{0 n}^{2} \operatorname{Im}(\langle 0|\mathbf{P} \cdot \mathbf{d}| n\rangle\langle n|\mathbf{D}| 0\rangle)}{E_{0 n}^{2}-\epsilon^{2}},
\end{aligned}
$$

where $\epsilon=\hbar \omega$ and $\mathbf{D}=-e \sum_{k=1}^{N_{e}} \mathbf{r}_{\mathbf{k}}$.

The energy-dependent factor may be presented as

$$
\frac{E_{0 n}^{2}}{E_{0 n}^{2}-\epsilon^{2}}=1+\frac{\epsilon^{2}}{E_{0 n}^{2}-\epsilon^{2}} .
$$

The energy-independent term 1 on the right-hand side allows us to sum over states $|n\rangle$ in Eq. (21). Using the closure condition and the commutator relation $[\mathbf{P}, \mathbf{D}]=$ $-i e \hbar N_{e}$, this term gives

$$
\begin{aligned}
\mathbf{D}_{\text {atom }}= & \mathbf{d}+\mathbf{D}_{\text {ind }}=\frac{Z_{i}}{Z} \mathbf{d} \\
& +\frac{2}{Z e \hbar} \sum_{n} \frac{\epsilon^{2} \operatorname{Im}(\langle 0|\mathbf{P} \cdot \mathbf{d}| n\rangle\langle n|\mathbf{D}| 0\rangle)}{E_{0 n}^{2}-\epsilon^{2}} .
\end{aligned}
$$

We observe that, in agreement with the Schiff theorem, the atomic electric dipole moment $\mathbf{D}_{\text {atom }}$ vanishes in a neutral atom $\left(Z_{i}=Z-N_{e}=0\right)$ with a static nuclear EDM $(\epsilon=\hbar \omega=0)$.

Assume that the nuclear EDM $d$ is directed along the $z$ axis. Using the nonrelativistic commutator relation $\mathbf{P}=-\frac{i m_{e}}{e \hbar}\left[H_{0}, \mathbf{D}\right]$ (where $m_{e}$ is the electron mass), we can express the atomic EDM in terms of the atomic dynamical polarizability $\alpha_{z z}(\omega)$

$$
\begin{aligned}
D_{\text {atom }}^{z} & =\frac{d_{z}}{Z}\left(Z_{i}-\frac{m_{e} \epsilon^{2} \alpha_{z z}}{e^{2} \hbar^{2}}\right), \\
\alpha_{z z} & =2 \sum_{n} \frac{E_{n 0}\left|\left\langle 0\left|D_{z}\right| n\right\rangle\right|^{2}}{E_{n 0}^{2}-\epsilon^{2}} .
\end{aligned}
$$

The axion field oscillation frequency may be very small on the atomic scale, and therefore, we may use static polarizabilities in this expression which are known for all atoms. The formula (24) may be rewritten, with the energy and the polarizability expressed in atomic units $\tilde{\epsilon}=\frac{\epsilon}{e^{2} / a_{B}}$ and $\tilde{\alpha}_{z z}=\frac{\alpha_{z z}}{a_{B}^{3}}$ (where $a_{B}$ is the Bohr radius), as

$$
D_{\mathrm{atom}}^{z}=\frac{Z_{i}-\tilde{\epsilon}^{2} \tilde{\alpha}_{z z}}{Z} d_{z}
$$

Since the atomic EDM $D_{\text {atom }}$ is proportional to $1 / Z$, it appears that the shielding is stronger in heavy atoms. 
This, however, is not necessarily the case since, for example in hydrogen and helium $\tilde{\boldsymbol{\alpha}}_{z z} \sim 1$ whereas $\tilde{\boldsymbol{\alpha}}_{z z} \sim$ 400 in cesium $(Z=55)$. Indeed, the numerical value of the polarizability $\tilde{\alpha}_{z z}$ in atomic units often exceeds the value of the nuclear charge $Z$, and therefore, the suppression of the $\mathrm{EDM}$ in a neutral atom mainly comes from the small frequency of the dark matter field oscillations in atomic units, $\tilde{\epsilon}$.

\section{OSCILLATING MOLECULAR EDMS INDUCED BY OSCILLATING NUCLEAR EDMs}

We see from the first line in Eq. (24) that the residual EDM in a neutral system $\left(Z_{i}=0\right)$ is proportional to the mass $m$ of the particle which produces the screening of the nuclear EDM d. The masses of nuclei $M_{N}$ in a molecule are up to 5 orders of magnitude larger than the mass of the electron $m_{e}$. In addition, the interval between molecular rotational energy levels ( $\sim m_{e} / M_{N}$ atomic units) are many orders of magnitude smaller than typical energy intervals in atoms and this may give an additional enormous advantage; see the denominator in the second line of Eq. (24). Finally, since the molecular spectra are very rich, the energy intervals are small and may be tuned by electric and magnetic fields, and it is easier to bring them into resonance with the small oscillation frequency of the axion dark matter field.

Calculations presented in the Supplemental Material [30] give the following results for the induced electric dipole of a neutral diatomic molecule when $\epsilon$ is smaller or of the order of the first rotational energy $E_{\text {rot }}$ :

$$
\mathbf{D}_{\mathrm{mol}}^{\mathrm{EDM}} \approx \frac{2 \mu_{N} \bar{X} \bar{d} E_{\mathrm{rot}}}{3 e \hbar^{2}} \frac{\epsilon^{2}}{E_{\mathrm{rot}}^{2}-\epsilon^{2}}\left(\frac{\mathbf{d}_{1}}{Z_{1}}-\frac{\mathbf{d}_{2}}{Z_{2}}\right)
$$

where $\mu_{N}=M_{1} M_{2} /\left(M_{1}+M_{2}\right)$ is the reduced nuclear mass, $\bar{X}$ is the ground-state internuclear distance, $\bar{d}$ is the ground-state intrinsic electric dipole of a polar molecule, $E_{\mathrm{rot}} \approx \hbar^{2} \mu_{N}^{-1} \bar{X}^{-2}$ is the energy of the first rotational state and $\mathbf{d}_{1,2}$ are the nuclear EDMs. In writing Eq. (26), we have assumed that the molecular ground state has a total angular momentum of 0 .

For $d_{1} \sim d_{2}$, we see that the lighter nucleus gives the dominant contribution. In other words, if $Z_{1} \ll Z_{2}$ then the term $\mathbf{d}_{2} / Z_{2}$ drops out. We assume this is the case. In the limits $\epsilon \ll E_{\text {rot }}$ and $\epsilon \gg E_{\text {rot }}$, Eq. (26) gives

$$
\frac{\mathbf{D}_{\mathrm{mol}}^{\mathrm{EDM}}}{\mathbf{d}_{1}} \approx \begin{cases}\frac{2 \epsilon^{2} \mu_{N}^{2} \bar{X}^{3} \bar{d}}{3 e \hbar^{4} Z_{1}} & \epsilon \ll E_{\mathrm{rot}}, \\ \frac{2 \bar{d}}{3 e Z_{1} \bar{X}} & \epsilon \gg E_{\mathrm{rot}} .\end{cases}
$$

We see that in the small-axion-mass limit $\left(\epsilon=m_{a} c^{2} \ll E_{\text {rot }}\right)$, heavy molecules have an advantage $\left(\mu_{N}^{2} / Z_{1}\right)$. In the large-axion-mass limit $\left(\epsilon=m_{a} c^{2} \gg E_{\mathrm{rot}}\right)$, the ratio of the EDMs is independent of $\epsilon$ and has an asymptotic value of $2 \bar{d} /\left(3 e Z_{1} \bar{X}\right)<2 /\left(3 Z_{1}\right) \leq 2 / 3(\bar{d} \sim e \bar{X}$
TABLE I. Position of the resonance (rotational or $\Omega$ doublet), large-axion-mass asymptotic and resonance values of the ratio $\left|D_{\mathrm{mol}}^{\mathrm{EDM}} / d_{1}\right|$ between the magnitude of the molecular EDM induced by the oscillating nuclear EDM $\mathbf{d}_{1}$ and $d_{1}$ in several molecules.

\begin{tabular}{lccc}
\hline \hline & $\begin{array}{c}\text { Resonance } \\
\text { position }(\mathrm{eV})\end{array}$ & $\begin{array}{c}\text { Large } \omega \\
\text { value }\end{array}$ & $\begin{array}{c}\text { Resonance } \\
\text { value }\end{array}$ \\
\hline $\mathrm{HF}\left({ }^{1} \Sigma^{+}\right)$ & $5.2 \times 10^{-3}$ & 0.8 & $8 \times 10^{5}$ \\
$\mathrm{LiF}\left({ }^{1} \Sigma^{+}\right)$ & $3.4 \times 10^{-4}$ & 0.2 & $2 \times 10^{5}$ \\
$\mathrm{YbF}\left({ }^{2} \Sigma^{+}\right)$ & $6.0 \times 10^{-5}$ & 0.04 & $4 \times 10^{4}$ \\
$\mathrm{BaF}\left({ }^{2} \Sigma^{+}\right)$ & $5.3 \times 10^{-5}$ & 0.02 & $2 \times 10^{4}$ \\
$\mathrm{TlF}\left({ }^{1} \Sigma^{+}\right)$ & $5.6 \times 10^{-5}$ & 0.06 & $6 \times 10^{4}$ \\
$\mathrm{HfF}^{+}\left({ }^{1} \Sigma^{+}\right)$ & $7.5 \times 10^{-5}$ & 0.04 & $4 \times 10^{4}$ \\
$\mathrm{HfF}^{+}\left({ }^{3} \Delta_{1}\right)$ & $4.1 \times 10^{-11}$ & 0.06 & $6 \times 10^{4}$ \\
$\mathrm{ThF}^{+}\left({ }^{1} \Sigma^{+}\right)$ & $5.8 \times 10^{-5}$ & 0.04 & $4 \times 10^{4}$ \\
$\mathrm{ThF}^{+}\left({ }^{3} \Delta_{1}\right)$ & $2.9 \times 10^{-10}$ & 0.06 & $6 \times 10^{4}$ \\
$\left.\mathrm{ThO}^{1}{ }^{1} \Sigma^{+}\right)$ & $7.6 \times 10^{-5}$ & 0.03 & $3 \times 10^{4}$ \\
$\mathrm{ThO}\left({ }^{3} \Delta_{1}\right)$ & $7.7 \times 10^{-10}$ & 0.05 & $5 \times 10^{4}$ \\
$\mathrm{WC}\left({ }^{3} \Delta_{1}\right)$ & $4.1 \times 10^{-12}$ & 0.08 & $8 \times 10^{4}$ \\
\hline \hline
\end{tabular}

for polar molecule) so molecules with at least one light nucleus are more advantageous.

The result (26) applies for the off-resonance case. If $\epsilon=E_{\mathrm{rot}}$ then we have the following relation between the oscillation amplitudes of $\mathbf{D}_{\mathrm{mol}}^{\mathrm{EDM}}$ and $\mathbf{d}_{1}$ :

$$
D_{\mathrm{mol}}^{\mathrm{EDM}} \approx \frac{2 \bar{d}}{3 e Z_{1} \bar{X}} \frac{E_{\mathrm{rot}}}{\Gamma} d_{1}
$$

which is the large-axion-mass asymptotic value in Eq. (27) multiplied by the resonance enhancement factor $E_{\text {rot }} / \Gamma$ where $\Gamma$ is the width. Again, we see that molecules with at least one light nucleus provide a bigger effect.

There may be different contributions to $\Gamma$, such as the natural width (which is typically small), Doppler width, collision width and time of flight (if the experiment is done

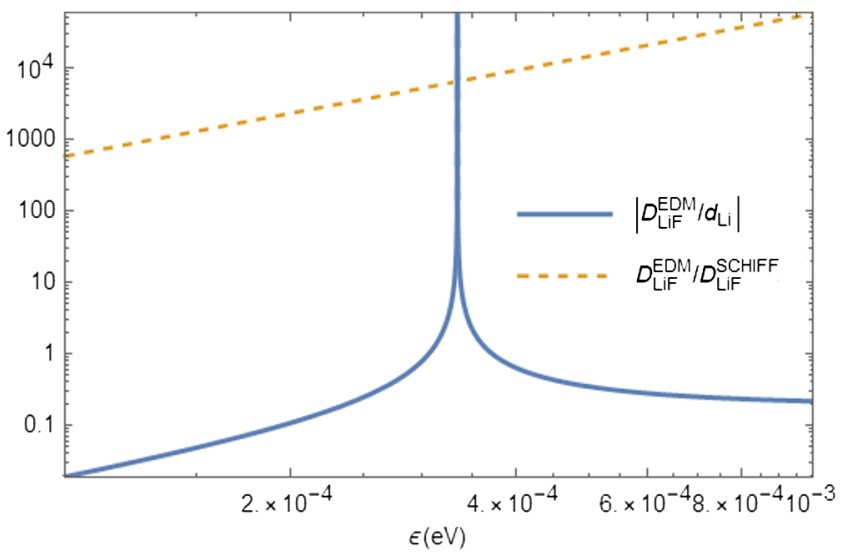

FIG. 1. Ratios of the molecular EDM induced by a nuclear EDM with the nuclear EDM and with the molecular EDM induced by a nuclear Schiff moment in LiF. 


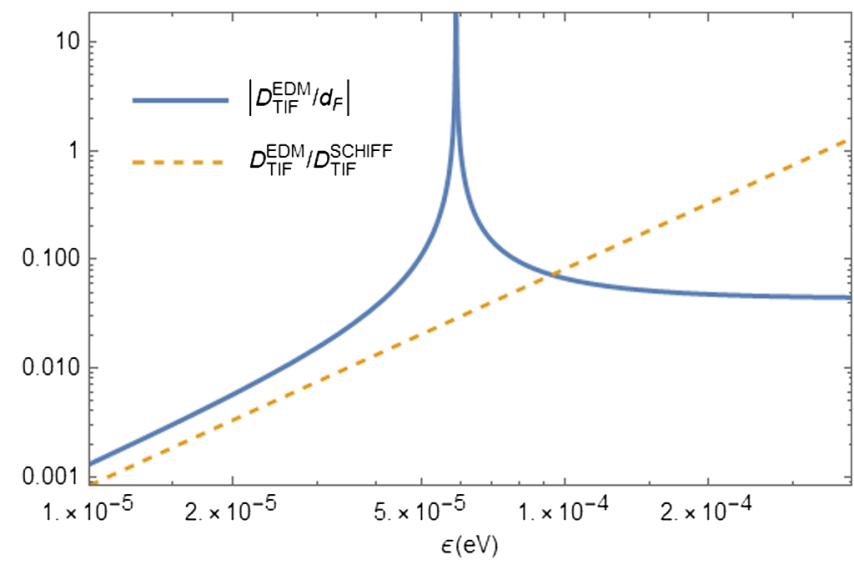

FIG. 2. Ratios of the molecular EDM induced by a nuclear EDM with the nuclear EDM and with the molecular EDM induced by a nuclear Schiff moment in TIF.

with a molecular beam). If, however, the experiment uses a trapped molecule then $\Gamma$ is mainly due to the velocity distribution of the axion: $\Gamma / E_{\mathrm{rot}} \approx\langle v\rangle^{2} / c^{2} \sim 10^{-6}$ where $\langle v\rangle$ is the mean axion velocity.

We remark that some molecules have ${ }^{3} \Delta_{1}$ as their ground or metastable state and thus have doublets of opposite parities and very small energy gaps (which may be manipulated by external electric and magnetic fields to scan for resonance with the axionic dark matter field). Accordingly, if the axion mass $\epsilon$ is of the order of these doublet splittings, the coefficient $2 / 3$ in the results (26)-(28) should be replaced by $1 / 2$ and the first rotational energy $E_{\mathrm{rot}}$ by the energy $E_{\mathrm{dbt}}$ of the ${ }^{3} \Delta_{1}$ doublet splitting. Examples of this type are presented in Table. I.

In Figs. 1 and 2, we show the behavior of $D_{\mathrm{mol}}^{\mathrm{EDM}} / d_{1}$ in $\mathrm{LiF}$ and $\mathrm{TlF}$ (the solid lines). For comparison, we also present the ratio $D_{\mathrm{mol}}^{\mathrm{EDM}} / D_{\mathrm{mol}}^{\mathrm{SCHIF}}$ between the molecular EDM induced by a nuclear EDM with that induced by a nuclear Schiff moment. The large- $\epsilon$ asymptotic value and resonance value of $D_{\mathrm{mol}}^{\mathrm{EDM}} / d_{1}$ and the position of the first resonances (rotation or doublet) in some example molecules are summarized in Table I. Note that we have assumed that $E_{\mathrm{rot}, \mathrm{dbt}} / \Gamma \approx 10^{6}$ (a trapped molecule, where $\Gamma$ is due to the axion velocity distribution).

\section{CONCLUSION}

We have presented in this paper the possibility of observing axion-induced oscillating nuclear EDMs with oscillating external electric fields. We found that if the frequencies of the external field and the nuclear EDM are the same then nuclear spin rotation appears with the rotation angle growing linearly with time. Moreover, if the two frequencies also equal some transition frequency in atoms or molecules then the effect is resonantly enhanced. We also presented the atomic and molecular EDMs induced by oscillating EDMs. Although these quantities do exist as expectation values of electric dipole operators, they do not produce nuclear spin rotation in a constant external electric field.

On the other hand, if the electric field is oscillating but the nuclear EDM is constant then there is nuclear spin rotation about the direction of the electric field. However, in this case, atomic and molecular EDMs remain zero since, in accordance with the Schiff theorem, a static nuclear EDM is completely shielded by electrons. Nevertheless, the rotation of the nuclear spin is observable because the nucleus carries a magnetic moment which produces a rotating magnetic field.

\section{ACKNOWLEDGMENTS}

This work is supported by the Australian Research Council, Gutenberg Fellowship and New Zealand Institute for Advanced Study. We thank Igor Samsonov and Oleg Sushkov for helpful discussions.
[1] P. W. Graham and S. Rajendran, Phys. Rev. D 84, 055013 (2011).

[2] L. I. Schiff, Phys. Rev. 132, 2194 (1963).

[3] P. G. H. Sandars, Phys. Rev. Lett. 19, 1396 (1967).

[4] E. A. Hinds and P. G. H. Sandars, Phys. Rev. A 21, 471 (1980).

[5] O. P. Sushkov, V. V. Flambaum, and I. B. Khriplovich, Zh. Exp. Teor. Fiz. 87, 1521 (1984) [Sov. Phys. JETP 60, 873 (1984)].

[6] V. V. Flambaum, I. B. Khriplovich, and O. P. Sushkov, Phys. Lett. 162B, 213 (1985).
[7] V. V. Flambaum, I. B. Khriplovich, and O. P. Sushkov, Nucl. Phys. A449, 750 (1986).

[8] I. B. Khriplovich, Parity Nonconservation in Atomic Phenomena (Gordon \& Breach, Amsterdam, 1991).

[9] N. Auerbach, V. V. Flambaum, and V. Spevak, Phys. Rev. Lett. 76, 4316 (1996).

[10] S. G. Porsev, J. S. M. Ginges, and V. V. Flambaum, Phys. Rev. A 83, 042507 (2011).

[11] V. V. Flambaum and A. Kozlov, Phys. Rev. A 85, 022505 (2012).

[12] P. G. H. Sandars, Phys. Lett. 14, 194 (1965). 
[13] V. V. Flambaum, Yad. Fiz. 24, 383 (1976) [Sov. J. Nucl. Phys. 24, 199 (1976)].

[14] O. P. Sushkov and V. V. Flambaum, Zh. Exp. Teor. Fiz. 75, 1208 (1978) [Sov. Phys. JETP 48, 608 (1978)].

[15] Y. V. Stadnik and V. V. Flambaum, Phys. Rev. D 89, 043522 (2014).

[16] D. Budker, P. W. Graham, M. Ledbetter, S. Rajendran, and A. O. Sushkov, Phys. Rev. X 4, 021030 (2014).

[17] C. Abel, N. J. Ayres, G. Ban, G. Bison, K. Bodek, V. Bondar, M. Daum, M. Fairbairn, V. V. Flambaum et al., Phys. Rev. X 7, 041034 (2017).

[18] V. V. Flambaum, Phys. Rev. A 98, 043408 (2018).

[19] V. V. Flambaum and I. B. Samsonov, Phys. Rev. A 98, 053437 (2018).

[20] H. B. Tran Tan, V. V. Flambaum, and I. B. Samsonov, Phys. Rev. A 99, 013430 (2019).

[21] V. Spevak, N. Auerbach, and V. V. Flambaum, Phys. Rev. C 56, 1357 (1997).
[22] V. V. Flambaum, A, Wickenbrock, and D. Budker, arXiV: 1909.04970.

[23] V. V. Flambaum, H. B. Tran Tan, A, Wickenbrock, and D. Budker, arXiV:1910.07705.

[24] A. Arvanitaki, S. Dimopoulos, and K. Van Tilburg, Phys. Rev. X 8, 041001 (2018).

[25] R. J. Crewther, P. di Vecchia, G. Veneziano, and E. Witten, Phys. Lett. B 91, 487 (1980).

[26] N. Yamanaka, B. K. Sahoo, N. Yoshinaga, T. Sato, and K. Asahi, B. P. Das. Eur. Phys. J 53, 54 (2017).

[27] W. C. Haxton and E. M. Henley, Phys. Rev. Lett. 51, 1937 (1983).

[28] V. V. Flambaum, D. DeMille, and M. G. Kozlov, Phys. Rev. Lett. 113, 103003 (2014).

[29] L. D. Landau and E. M. Lifshitz, Quantum Mechanics (Pergamon Press, Oxford, 1965).

[30] See Supplemental Material at http://link.aps.org/supplemental/ 10.1103/PhysRevD.100.111301 for detailed calculation of molecular EDMs induced by oscillating nuclear EDMs. 